\title{
FIRST ORDER VARIATION OF THE DISPERSION FUNCTION WITH PARTICLE ENERGY DEVIATION ${ }^{\star}$
}

\author{
J. P. Delahaye ${ }^{\mathfrak{S}}$ and J. Jäger \\ Stanford Linear Accelerator Center \\ Stanford University, Stanford CA 94905
}

\section{Summary}

The variation of the dispersion fonction with the particle energy deviation can presently be calculated from second order transfer matriees $(1,2)$; its periadic solution is det srmined numerically.

The general differential equations for the dispersion function deduced from the complete equation of motion to second order ${ }^{(9)}$ are solved, using Green's function in'egral leading to an analytital expresioion ${ }^{(4)}$ of the periodic solution of the dispersion function $D_{0}$ and of the first order perturbation, $D_{1}$, with respect to energy deviation. The same method can be extended to higher order perturbations of the dispersion function.

The determination of the periodic solution as well as the traneportation of these two dispersion funetions through any element depends only on two particular integrals. These integrala are derived for the general case of a combined function magnet, with up to second order components. The derivation includes the contribution from the edges. Chapter 2 and 3 deal with closed machines, chapter 4 applies these results to beam transport lines.

These aralytical expressions are then applied to a typical machine in order to illustrate the most important driving terms; the results do agree with those obtained by optics programs like MAD(1) or DIMAT(2) based on second order transfer matrices.

- Work supported by the Department of Energy, contract DE-AC03-76SF00515.

$\$$ Permanent address: CERN, Geneva 23, Switzeriand.

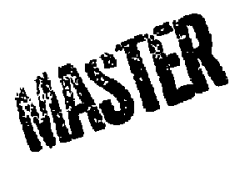




\section{Differential equation for the Dispersion function}

The differential equation of particle motion in the horisontal plane in the variablea $x, y$ and their derivatives to second order can be expressed as follows $(9)$;

$$
\begin{aligned}
x^{\prime \prime}-h(1+h x)-x^{\prime}\left(h x^{\prime}+x h^{\prime \prime}\right)=\left(1-\delta+\delta^{2}\right)[-h & +\left(k-2 h^{2}\right) x \\
& +\left(2 h k-h^{3}+\frac{1}{2} r\right) x^{2}-\frac{1}{2} h x^{\prime 2} \\
& \left.+\frac{1}{2}\left(h^{\prime \prime}-h k-r\right) y^{2}+1-y y^{\prime}-\frac{1}{2} h y^{\prime 2}\right]
\end{aligned}
$$

where the prime indicatea the derivative with reapect to the arimuth $s$ and

$\begin{array}{ll}x & \text { is the horinontal coordinate, } \\ y & \text { is the vertical coordinate, } \\ & \text { is the arc length along the reference orbit, } \\ h(s)=\frac{1}{\rho} & \text { is the curvature of the reference orbit, } \\ k(s)=-\left.\frac{1}{B_{p}} \frac{\partial B_{y}}{\partial x}\right|_{x=0} & \text { is the normalized quadrupole atrength, } \\ r(s)=-\left.\frac{2}{B_{p}} \frac{J^{2} B_{y}}{\partial x^{2}}\right|_{x=0} & \text { is the normalized zextupole component, } \\ B \rho & \text { is the particle rigidity, } \\ \delta=\frac{\Delta P}{P} & \text { is the particle energy deviation. }\end{array}$

The horisontal motion $x$ of a particle with energy deviation $\delta$ can be written in the form

$$
x=2+D \delta
$$

where $I$ is the betatron oscillation and $D \delta$ is the closed orbit of this particle. One derives the differential equation for the dispersion function $D$ (s) (also casled $\alpha_{p}$ or $\eta$ in accelerator theory) by inserting eq. (2.2) with $z \equiv 0$ into eq. (2.1). To first order in 6 one has

$$
D^{\prime \prime}+\left(h^{2}-k\right) D=h+\left[-h+\left(2 h^{2}-k+h^{\prime} D^{\prime}\right) D+\left(2 h k-h^{3}+\frac{1}{2} r\right) D^{2}+\frac{1}{2} h D^{\prime 2}\right] \delta .
$$

The expansion of the dispersion function in the form

$$
D=D_{0}+D_{\mathrm{t}} \delta
$$

enables to solve eq. (2.3) successively for each power of $\delta$. The disperaion function $D_{0}(s)$ is the 
periodi: solution of the well known differential equation

$$
D_{0}^{\prime \prime}+\left(h^{2}-k\right) D_{0}=h
$$

The differential equation for the perturbation to the first order in $\delta, D_{1}(s)$, in the periodic solution of

$$
D_{1}^{\prime \prime}+\left(h^{2}-k\right) D_{1}=-h+\left(2 h^{2}-k+h^{\prime} D_{0}^{\prime}\right) D_{0}+\left(2 h k-h^{3}+\frac{1}{2}+\right) D_{0}^{2}+\frac{1}{2} h D_{0}^{\prime 2} .
$$

In a series of reports $(4,5,0)$ the equation for $D_{1}$ has been derived for machines with large bending radius $\rho$ where higher order terms in $h$ as well as the terms coming from combined function magnets $(h k)$ and from the variation of the dispersion function with asimuth $\left(D_{0}^{j}\right)$ were disegarded. For small machines these terms cannot be neglected.

\section{The periodic solution}

The two differential equations $(2.5,2.6)$ for $D_{0}$ and $D_{1}$ are of the form

$$
D_{i}^{\prime \prime}+\left(h^{2}-k\right) D_{i}=f_{i} \quad \text { with } \quad i=0,1
$$

where

$$
\begin{aligned}
& f_{0}=h, \\
& f_{2}=-h+\left(2 h^{2}-k+h^{\prime} D_{0}^{\prime}\right) D_{0}+\left(2 h k-h^{3}+\frac{1}{2} r\right) D_{0}^{2}+\frac{1}{2} h D_{0}^{\prime 2} .
\end{aligned}
$$

Equation (3.1) is also valid for higher order perturbations of the disperaion function with more complicated driving term functions $f_{i}, i \geq 2$ in the r.h.s. of this equation.

Assuming that the Twiss parameters $(\beta, \alpha)$ and the phase advance $(\mu)$ along the central trajectory are known, using the usual variable transformations $(7)$

$$
\begin{aligned}
\phi(s) & =\int \frac{d s}{Q \beta}, \\
E_{i}(\phi) & =\frac{D_{i}}{\sqrt{\beta}}
\end{aligned}
$$

whete $Q$ is the horisontal tane (the number of horisontal betatron oseillations per turn around 
the machine) and taking into account the well known relation (7)

$$
\left(h^{2}-k\right) \beta^{2}+\frac{1}{2} \beta \beta^{\prime \prime}-\frac{1}{4} \beta^{\prime 2}=1
$$

the geaeral differential equation (3.1) for the dispersion function can be transformed into the equation of a forced harmonic oscillator:

$$
\frac{d^{2} E_{i}}{d \phi^{2}}+Q^{2} E_{i}=Q^{2} \beta^{3 / 2} J_{i}(\phi)
$$

where $f_{i}$ are the functions defined in eg. (3.2).

\subsection{HARMONIC ANALYSIS METHOD}

We shall we the Fourier analysis method to solve eq. (3.3). Since $\beta$ as well as $f_{i}(\phi)$ are periodic functione with the period $2 \pi$, they can be expanded into Fourier series resulting in the periodic solutions:

$$
D_{i}(s)=\sqrt{\beta(s)}\left[a_{i 0}+\sum_{p=1}^{\infty} \frac{Q^{2}}{Q^{2}-p^{2}}\left(a_{i p} \cos p \phi+b_{i p} \sin p \phi\right)\right]
$$

with

$$
\begin{aligned}
& a_{i 0}=\frac{1}{2 \pi} \int_{0}^{2 \pi} f_{i}(\phi) d \phi, \\
& a_{i p}=\frac{1}{\pi} \int_{0}^{2 \pi} f_{i}(\phi) \cos \phi \phi d \phi, \\
& b_{i p}=\frac{1}{\pi} \int_{0}^{2 \pi} f_{i}(\phi) \sin \phi \phi d \phi .
\end{aligned}
$$

If the horisontal tune $Q$ approaches an integer, the factor $Q^{2} /\left(Q^{2}-p^{2}\right)$ increases to infinity. As a consequence the Fourier components for the harmonics which are close to the horizontal betatron frequency $Q$ are drastically magnified by this factor. But this does not mean that only these ha monics will contribute to the gam in eq. (3.4). Depending on the element (quadrupoles, bending magnets and sextupoles) distribution in the machine some other harmonics of the function $f_{i}(\phi)$ can become dominant. 
T!: : harmonic analysis method is very useful to determine the driven harmonics ${ }^{(0)}$ numerically as the summation over $p$ in eq. (3.4) will vary from machine to machine, but it cannot be used to derive closed analytical expressions of the dispersion function.

\subsection{GREen FUNGTJON METHOD}

The periodit solution of the goneral difforential equation (3.3) can, by meang of Green's function, be written in the form ${ }^{(7)}$

$$
E_{i}(\phi)=\frac{Q}{2 \sin \pi Q} \int_{\phi}^{2 \pi+\phi} \beta^{3 / 2} f_{i}(\psi) \cos |Q(\pi+\phi-\psi)| d \psi
$$

Introducing the original variables

$$
\begin{aligned}
d \sigma & =Q \beta d \psi, \\
\mu(s) & =Q \phi, \\
D_{i}(s) & =E_{i} \sqrt{\beta},
\end{aligned}
$$

into eq. (3.5) gives

$$
D_{i}(s)=\frac{\sqrt{\beta(s)}}{2 \sin \pi Q} \int_{0}^{++L} \sqrt{\beta(\sigma)} f_{i}(\sigma) \cos [Q \pi+\mu(s)-\mu(\sigma)] d \sigma
$$

where $f_{i}$ are the functions defined in eq. (3.2) and where $L$ is the length (circumference) of the reference orbit. Diferentiating eq. (3.7) with respect to s leads to the periodic solution for $D^{\prime}(s)$ :

$$
D_{i}^{\prime}(s)=-D_{i}(s) \frac{\alpha(s)}{\beta(s)}-\frac{1}{2 \sqrt{\beta(s) \sin \pi Q}} \int_{s}^{\rho+L} \sqrt{\beta(\sigma)} f_{i}(\sigma) \sin [Q \pi+\mu(s)-\mu(\sigma)\} d \sigma .
$$

\section{Integration through the various elemente}

Actually, the integrals in eqs. (3.7), (3.8) can be replaced by aums of integrals over the varions magnetic elements which are ch:aracteizzed in the function $f_{i}(\sigma)$ by the terms containing

$$
h(h k) \ldots \text { for (combined function) bending magnets, }
$$




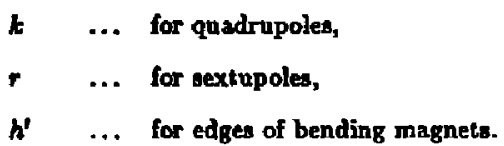

Assuming the hard edge approximation, where the magnetic field rises abruptly from zero outside the magnet to a constant value inside it, we can solve the integral on the r.h.s. of the eqs. (3.7), (3.8) for theme elements analytically.

Defining an element $j$ of length $l_{j}$ by its tranafer matrix

$$
\mathbf{R}_{\mathrm{j}}(\sigma)=\left(\begin{array}{ccc}
\boldsymbol{R}_{11} & \boldsymbol{R}_{13} & \boldsymbol{R}_{1 \mathrm{~s}} \\
\boldsymbol{R}_{21} & \boldsymbol{R}_{22} & \boldsymbol{R}_{23} \\
0 & 0 & 1
\end{array}\right)
$$

and asirg the values for the functions $\beta_{j}, \alpha_{j}$ and $\mu_{j}$ at the entrance of this element, the variation of the phase $\mu$ and the $\beta$-function are given by

$$
\begin{aligned}
& \mu(\sigma)=\arctan \left(\frac{R_{12}(\sigma)}{\beta_{j} R_{11}(\sigma)-\alpha_{j} R_{12}(\sigma)}\right) \\
& \beta(\sigma)=\beta_{j} R_{11}^{2}(\sigma)-2 \alpha_{j} \bar{R}_{12}(\sigma) R_{12}(\sigma)+\frac{1+\alpha_{j}^{2}}{\beta_{j}} R_{12}^{2}(\sigma) .
\end{aligned}
$$

Taking the origin $s=0$ as the starting point for the phase $\mu(s)$ and using identities for the inverse trigonometric functions, like

$$
\arccos x=\arctan \frac{\sqrt{1-z^{2}}}{z},
$$

one obtains

$$
\begin{aligned}
& \sqrt{\beta(\sigma)} \cos \left[\pi Q+\mu(s)-\mu_{j}-\mu(\sigma)\right]=\left[\sqrt{\bar{\beta}_{j}} \pi_{11}(\sigma)-\frac{\alpha_{j}}{\sqrt{\beta_{j}}} R_{12}(\sigma)\right] \cos \Delta \mu_{j}+\frac{R_{12}(\sigma)}{\sqrt{\beta_{j}}} \sin \Delta \mu_{j}, \\
& \sqrt{\beta(\sigma)} \sin \left[\pi Q+\mu(s)-\mu_{j}-\mu(\sigma)\right]=\left[\sqrt{\beta_{j}} R_{11}(\sigma)-\frac{\alpha_{j}}{\sqrt{\beta_{j}}} R_{12}(\sigma)\right] \sin \Delta \mu_{j}-\frac{R_{12}(\sigma)}{\sqrt{\beta_{j}}} \cos \Delta \mu_{j},
\end{aligned}
$$

where

$$
\begin{array}{ll}
\Delta \mu_{j}=\pi Q+\mu(s)-\mu_{j} & \text { for } \mu(s)<\mu_{j}, \\
\Delta \mu_{j}=-\pi Q+\mu(s)-\mu_{j} & \text { for } \mu(s)>\mu_{j} .
\end{array}
$$


Thus eqa. (3.7), (3.8) can be written in the following form:

$$
\begin{aligned}
& D_{i}(s)=\frac{\sqrt{\beta(\sigma)}}{2 \sin \pi Q} \sum_{i}\left\{\sqrt{\beta_{j}} \cos \Delta \mu_{j} \int_{0}^{l} f_{i}(\sigma) R_{11}(\sigma) d \sigma\right. \\
& \left.-\frac{1}{\sqrt{\beta_{j}}}\left(a_{j} \cos \Delta \mu_{j}-\sin \Delta \mu_{j}\right) \int_{0}^{l_{j}} f_{i}(\sigma) R_{12}(\sigma) d \sigma\right\}, \\
& D_{i}^{\prime}(s)=-D_{i}(s) \frac{\alpha(s)}{\beta(s)}-\frac{1}{2 \sqrt{\beta(\theta) \sin \pi Q}} \sum_{j}\left\{\sqrt{\beta_{j}} \sin \Delta \mu_{j} \int_{0}^{l} f_{i}(\sigma) R_{11}(\sigma) d \sigma\right. \\
& \left.-\frac{1}{\sqrt{\beta_{i}}}\left(\alpha_{j} \sin \Delta \mu_{j}+\cos \Delta \mu_{j}\right) \int_{0}^{I_{j}} f_{i}(\sigma) R_{10}(\sigma) d \sigma\right\} \text {, }
\end{aligned}
$$

where $j$ runs over the elements of the machine. These expressions were already derived(4) for the perturbation $D_{1}$. In fact, eqs. (3.11) are valid for any order of the expansion of the disperaion function. Their periodic solutions are completely determined by the following integrals

$$
\int_{0}^{1} f_{i}(\sigma) R_{11}(\sigma) d \sigma \quad \text { and } \quad \int_{0}^{l} f_{i}(\sigma) R_{12}(\sigma) d \sigma
$$

over the elements for which the functiong $f_{i}$ are different from cero.

Equations (3.2) shows that the function $D_{0}$ is only driven by the bending magnets $(h \neq 0)$ while the function $D_{1}$ is driven by the combined function bendirg magnets $(h k \neq 0)$, their edges $\left(h^{\prime} \neq 0\right)$ and by the quadrupoles $(k \neq 0)$ and the sextupoles $(r \neq 0)$.

The computation of the above integrals is derived in APPENDIX I for all elements up to second order by replacing the transfer matrix elements by their corresponding values. 


\section{Transport of the Dispersion function}

Although the equations (9.11) define the periodic solution of $D_{i}$ and $D_{i}^{\prime}$ respectively every* where in the machine, it is important to know the variation of $D_{1}$ and $D_{i}^{\prime}$ dye to the different: elements in a ring. Moreover, this facilitates the calculation of the perturbation of the diapersion function slong transfer lines.

Supposing the values of the Dispersion functions $D_{i}$ and their derivatives $D_{i}^{\prime}$ are known at the point $s_{D}$ either by determination of the periodic solution in a ring using eqa. (3.11) or as given initial values for a transfer line, the transported values of the functions $D_{i}$ and $D_{i}^{\prime}$ can than be determined at any point $s$ of the transfer channnel using the transfer matrix $M$ defined as

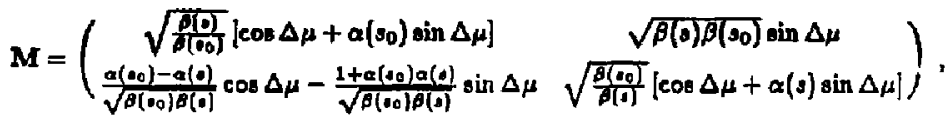

with $\Delta \mu=\mu(s)-\mu\left(s_{0}\right)$.

In fact, the general solution of eq. (3.3) can be written in the form

$$
E_{i}(\phi)=A_{i} \cos \left[Q\left(\phi-\phi_{0}\right)\right]+B_{i} \sin \left[Q\left(\phi-\phi_{0}\right)\right]+Q \int_{\phi_{0}}^{\phi} \beta^{s / 2} f_{i}(\psi) \sin \left[Q\left(\phi-\phi_{0}-\psi\right)\right] d \psi
$$

where $\phi \equiv \phi(s)$ and $\phi_{0} \equiv \phi\left(s_{0}\right)$ and with the coeffients $A_{i}, B_{i}$ determined by the initial conditions:

$$
A_{i}=E_{i}\left(\phi_{0}\right), \quad B_{i}=\frac{1}{Q} \frac{d E_{i}\left(\phi_{0}\right)}{d \phi}
$$

Using the original variables as defined in eq. (3.6), the Dispersion funetions at any point a are defined by

$$
D_{i}(s)=D_{i}\left(s_{0}\right) M_{11}+D_{i}^{t}\left(s_{0}\right) M_{12}+\sqrt{\beta(\Delta)} \int_{0_{0}}^{1} \sqrt{\beta(\sigma)} f_{i}(\sigma) \sin \left[\mu(s)-\mu\left(s_{0}\right)-\mu(\sigma)\right] d \sigma
$$

Hence, the dispersion functiona can be determined successively from the entry (beginning) $D_{i}(0)=D_{i b}$ to the exit $D_{i}(l)$ of each element with $\mathrm{M}=\mathrm{R}(l)$ w the tranger matrix of this 
particular element. Introducing eq. (3.10) into eq. (4.1) simplifes the equations for the diopersion functions to

$$
\begin{aligned}
& D_{i}(l)=D_{i b} M_{11}+D_{i b}^{\prime} M_{12}+M_{12} \int_{0}^{l} f_{i}(\sigma) R_{11}(\sigma) d \sigma-M_{11} \int_{0}^{l} f_{i}(\sigma) R_{12}(\sigma) d \sigma, \\
& D_{i}^{\prime}(l)=D_{i b} M_{21}+D_{i b}^{\prime} M_{22}+M_{22} \int_{0}^{l} f_{i}(\sigma) R_{11}(\sigma) d \sigma-M_{21} \int_{0}^{l} f_{i}(\sigma) R_{12}(\sigma) d \sigma .
\end{aligned}
$$

The transport of the Dispersion function $D_{0}$ is easy to deduce from the transfer matrix coefficients:

The introduction of

$$
\begin{aligned}
& \int_{0}^{l} h\left[M_{12} R_{11}(\sigma)-M_{11} R_{12}(\sigma)\right] d \sigma=M_{19}, \\
& \int_{0}^{l} h\left[M_{22} R_{11}(\sigma)-M_{21} R_{12}(\sigma)\right] d \sigma=M_{29}
\end{aligned}
$$

into eq. (4.2) leads to the well known formulae

$$
\begin{aligned}
& D_{0}(l)=D_{08} M_{11}+D_{06}^{\prime} M_{12}+M_{13}, \\
& D_{0}^{\prime}(l)=D_{0 b} M_{91}+D_{06}^{\prime} M_{22}+M_{3 s} .
\end{aligned}
$$

The transport of the Dispersion function $D_{1}$ uses the same integrals (3.12) as those needed for the calculation of its periodic solution; they are listed in APPENDDX I.

The complete expressions for the transport of the function $D_{1}$ through the different machine elements are summarised in APPENDIX II. 


\section{Application}

Tho LEP Electron Positron Accurmulator $\left(\right.$ EPA) ${ }^{(9)}$ provides an ideal application for the calculation of the Disperzion function $D_{1}$. In fact, in this machine all the driving terms of this function are present becanse of the low bending radius $(\rho=1.42 B \mathrm{~m})$ and of its combined function bending magnets $\left(k=0.5 \mathrm{~m}^{-2}\right)$ with entrance and exit angles.

Moreover, the strong sextupoles $\left(r= \pm 8.25 \mathrm{~m}^{-3}\right)$ localized in the arce do perturb the Dispervion fanction $D_{1}$ in the lene dispernion free sections $\left(D_{0}=0\right)$.

The results calculated for one superperiod of EPA maing the program COMFORT ${ }^{(10)}$ are displayed in the following table as well as plotted in fig. 1 and fig. 2 for the cases without and with the sextupoles reapectively to demonstrate their driving force.

\section{Acknowledgements}

'Ve would like to take the opportunity to thank the members of the Beam Dynamics Group for their belpful and encouraging diseussions. In particular, we want to thank R. Servranckx who helped us to clarify some difficult points in second order optics. 
sextupoles OFF

$\begin{array}{ll}\text { elen } & B[\mathrm{~m}] \\ \text { BEO } & 0.00000\end{array}$

D1

2. 02850

DFWH $\quad 3.11850$

QFUH

3. 30860

D2

EDOE

B

EDGE

D3

sV

D2

EDEE

B

EDGE

D2

QFLH

QFLH

DS

SXH

SXH

D5

QRLE

QFLB

D2

EDEE

B

EDGE 10.57950

D2

SV

D3

EDEE

B

13.56950

BFWH 14.02150

QFWH 14.40150

D1 $\quad 17.33000$

D6 18.07160

QFNH 18.26650

DFNH 18.46150

D7 20.82400

ADNB 21.01900

PDNH 21.21400

D8 22.02560

DB 22.83700

ADKH 23.03199

\begin{abstract}
Do $[m]$
\end{abstract}
0.00000

0.00000

0.00000

0.00000

0.00000

0.00000

0.10998

0.10998

0.78679

0.90925

1.09785

1.09786

1.62373

1. 52373

2.06545

2.23468

2.29205

2. 29205

2. 29205

2.29204

2. 29204

2.23468

2.06546

1.52373

1.62373

1.09784

1.0.2784

0.90924

0.78678

0.10996

0.10996

$-0.00003$

$-0.00003$

$-0.00004$

$-0.00004$

$-0.00004$

$-0.00003$

$-0.00003$

$-0.00003$

$-0.00003$

$-0.00001$

$-0.00001$

$-0.00001$

0.00000

0.00001

0.00001

\begin{abstract}
0.00000
\end{abstract}
0.00000

0.00000

0.00000

0.00000

0.00000

$0.3928 \mathrm{~T}$

0.40821

0.40821

0.40821

0.40821

0.56134

0.96001

1. 17255

1. 17266

0.60133

0.00000

0.00000

0.00000

0.00000

0.00000

$-0.60133$

$-1.17266$

$-1.17266$

$-0.96002$

$-0.66136$

$-0.40821$

$-0.40821$

$-0.40821$

$-0.40821$

$-0.39288$

$-0.00001$

$-0.00001$

$-0.00001$

$-0.00001$

0.00000

0.00000

0.00000

0.00001

0.00001

0.00001

0.00001

0.00001

0.00001

0.00001

0.00001

D1 [m]

$-0.63777$

$-0.63777$

$-0.62409$

$-0.68716$

$-0.56678$

$-0.46576$

$-0.46268$

$-0.46262$

$-0.94178$

$-1.02860$

$-1.16205$

$-1.17877$

$-1.24345$

$-1,21124$

$-1.93352$

$-2.12636$

$-2.10136$

$-1.84216$

$-1.79490$

$-1.62766$

$-1.3684$

$-1.14235$

$-0.74768$

0.38676

0.36465

1.06889

1.08561

1.80390

2. 41875

6. 31947

6. 31931

6. 69377

6. 69377

8. 39798

8.47106

6.97107

6. 69127

6. 42662

6.13502

1.85404

1.61800

1.21224

$-0.00585$

$-1.22394$

$-1.62982$
7.98836
01 '

0.00000

0.00000

0.13407

0.26277

0.26277

0.19780

$-0.17646$

$-0.28906$

$-0.28906$

$-0.28906$

$-0.28906$

$-0.27957$

0.12438

$-1,56338$

$-1.66338$

$-0.46044$

0.71503

0.71503

0.71609

0.71609

0,71603

1.65243

2.47719

2.47713

1.01366

1. 42651

1,74053

1.74953

1.74953

1.74953

2.44346

2. 08884

3.01859

3.01869

1. 27882

$-0.51220$

$-0.51220$

- $\$ 1220$

$-1.11372$

$-1.81206$

$-1,81206$

$-1.64019$

$-1.50169$

$-1.50108$

$-1,50103$

$-1.64130$

sextupoles ON

$$
\begin{aligned}
& r_{m v}=8.25 \mathrm{~m}^{-3} \\
& r_{\text {sxh }}=-8.95 \mathrm{~m}^{-.3}
\end{aligned}
$$

\section{DI [n] \\ D1 *}

2.39710

2. 39719

2.34916

2. 20598

1. 75068

1. 75068

1.22930

1. 22947

$-0.40414$

$-0.67243$

$-0.61677$

$-0.63249$

$-0.54438$

$-0.51217$

$-1.06267$

$-1.20723$

$-1.17998$

$-0.96081$

$-1.11687$

$-1.76042$

$-9.93240$

$-4.00277$

$-5.51694$

$-6.65110$

$-6.68330$

$-9.07381$

$-0.05700$

$-11.54779$

$-13.02499$

$-20.48546$

$-20.48563$

$-24.76210$

$-24.76218$

$-29.99934$

$-31.53763$

$-31.81204$

$-26.17886$

$-24.75252$

$-24.13410$

$-23.03914$

$-6.96244$ 


\begin{tabular}{|c|c|c|c|c|c|c|c|}
\hline QDHA & 23.22690 & 0.00001 & 0.00001 & -1.86620 & -1.81448 & 7.00865 & j.81412 \\
\hline D7 & 26.68949 & 0.00003 & 0.00001 & -6.16291 & -1.81448 & 23.10691 & 6.81412 \\
\hline 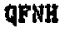 & 25.78449 & 0.00005 & 0.00001 & -6.44480 & $-1,17491$ & 24.20305 & 4.41002 \\
\hline QENH & 25,97949 & 0.00003 & 0.00000 & -6.60938 & -0.51096 & 24.82116 & 1.91881 \\
\hline D6 & 26.72099 & 0.00003 & 0.00000 & -6.98825 & -0.61095 & 36.24396 & 1. 91881 \\
\hline D6 & 27.46249 & $0.0000 \Omega$ & 0.00000 & -7.36712 & $-0 . \overline{1} 1095$ & 27.66676 & 1. 91881 \\
\hline QFHH & 27.65749 & 0.00003 & 0.00000 & -7.39366 & 0.23014 & 27.76644 & -0.89807 \\
\hline QENH & $2 i .85249$ & 0.00003 & 0.00000 & -7.27416 & 0.98450 & 2731766 & -3.69722 \\
\hline D7 & 30.21499 & 0.00002 & 0.00000 & -4.94827 & 0.98450 & 18.68298 & -3.69722 \\
\hline QDHH & 30.40999 & 0.00002 & 0.00000 & -4.80500 & 0.48739 & 18.04493 & -1.83036 \\
\hline QDNH & 30.60499 & 0.00002 & 0.00000 & -4.76756 & 0.00000 & $1 T .86677$ & 0.00000 \\
\hline DB & 31.41649 & 0.00002 & 0.00000 & -4.76756 & 0.00000 & 17. B6677 & 0.00000 \\
\hline D8 & 32.22799 & 0.00092 & 0.00000 & -4.76756 & 0,00000 & 17.86677 & 0.00000 \\
\hline QDNA & 32.42299 & 0.00002 & 0.00000 & -4.80500 & -0.48739 & 18.04493 & i. .83036 \\
\hline qDNH & 32.61799 & 0.00002 & 0.00000 & -4.94827 & -0.98450 & 18. 68298 & 3.69722 \\
\hline D7 & 34.98049 & 0.00003 & 0.00000 & -7.27416 & $-0.9 B 450$ & 27.31766 & ง. 69722 \\
\hline QFNH & 35,17548 & 0.00003 & 0.00000 & -7.39366 & -0.23914 & 27.76644 & 0. B9807 \\
\hline QFNH & 36.37049 & 0.00003 & 0.00000 & -7.36712 & 0.61095 & $27.6667 f$ & -1.01881 \\
\hline D6 & 36.11100 & 0.00003 & 0.00000 & -6.98826 & 0.51085 & 26.24396 & -1.9188 \\
\hline D6 & 36.86349 & 0.00003 & 0.00000 & -6.60938 & 0.51085 & 24.82116 & -1.91861 \\
\hline AFNH & 97.04849 & 0.00003 & -0.00001 & -6.44480 & 1.17431 & 24.20308 & -2.41002 \\
\hline QRHH & 37.24349 & 0.00003 & -0.00001 & -6.15291 & 1.81448 & 23.10691 & -6.81412 \\
\hline bt & 39.60599 & 0.00001 & -0.00001 & -1.86620 & 1.81448 & 7.00865 & -6.81412 \\
\hline QDNH & 39.80059 & 0.00001 & $-0,00001$ & -1.52982 & 1.64139 & 5.74527 & $-6.1640 B$ \\
\hline QDHH & 39.99599 & 0.00001 & -0.00001 & -1.22394 & 1.50103 & 4.69657 & -5.63698 \\
\hline eg & 40.80749 & 0.00000 & -0.00001 & -0.00585 & 1.60103 & 0.02216 & -5.63698 \\
\hline D8 & 41.61899 & -0.00001 & -0.00001 & 1.21224 & 1.60103 & -4.65224 & -5.63698 \\
\hline QDARE & 41.81399 & -0.00001 & -0.00001 & 1.51800 & 1.64019 & -5.70050 & -6.15954 \\
\hline QDNH & 42.00899 & -0.00001 & -0.00001 & 1.85404 & 1.81206 & -6.96244 & -6.80495 \\
\hline D7 & 44.37149 & -0.00003 & -0.00001 & 6.13502 & 1.81206 & -23.03914 & -6.80495 \\
\hline QFNH & 44.56649 & -0.00003 & -0.00001 & 6.42662 & 1.17372 & -24.13419 & -4.40780 \\
\hline QFNH & 44.76149 & -0.00003 & 0,00000 & 6.69127 & 0.51220 & -24.75252 & -1.92367 \\
\hline D6 & 45.50299 & -0.00003 & 0.00000 & 6.97107 & 0.61220 & -26.17886 & -1.92357 \\
\hline 91 & 48.43149 & -0.00004 & 0.00000 & 8.47106 & 0.61220 & -31.81204 & -1.92367 \\
\hline QFWH & 48.62149 & -0.00004 & 0.00001 & 8. 3979B & $-1,27882$ & -31.63763 & 4.80236 \\
\hline QFYH & 48.81149 & -0.00004 & 0.00001 & 7.98836 & -3.01859 & -29.99934 & 11.33684 \\
\hline D2 & 49.27349 & -0.00003 & 0.00001 & 6.69377 & -3.01859 & -24.76219 & 11.33584 \\
\hline EDAE & 49.27349 & -0.00003 & 0.00001 & 6.69377 & -2.09884 & -24.76219 & 7.88184 \\
\hline B & 49.83349 & 0.10996 & 0.39288 & 5.31931 & -2.44345 & -20.48563 & 7.40622 \\
\hline EDGE & 49.83349 & 0.10996 & 0.40821 & 5.31947 & -1.74963 & -20.48546 & 4.49968 \\
\hline D3 & E1. 49149 & 0.78678 & 0.40821 & 2.41876 & -1.74953 & $-13.0240 y$ & 4.49968 \\
\hline 8v & 51.79149 & 0.90924 & 0.40821 & 1.89390 & -1.74963 & -11.54779 & Б. 39114 \\
\hline D2 & 62.26349 & 1.09784 & 0.40921 & 1.08561 & -1.74853 & -9.05709 & 5. 39114 \\
\hline BDGE & 52.25349 & 1.09784 & 0.66135 & 1.06889 & -1.42651 & -9.07381 & $4.2893 \mathrm{E}$ \\
\hline$B$ & 62.81349 & 1.52373 & 0.06002 & 0.36455 & -1.01366 & -5.58339 & 4.67276 \\
\hline BDOE & 52.81349 & 1.52373 & 1.17256 & 0.39676 & -2.47713 & -6.66119 & 2.24014 \\
\hline 12 & 53.27549 & 2.06546 & 1.17256 & -0.74768 & -2.47713 & -5.61624 & 2.24014 \\
\hline QFLH & 63.46549 & 2.23468 & 0.60133 & -1.14235 & -1.65243 & -4.90877 & 4.19783 \\
\hline QFLK & 63.65649 & 2.20204 & $\$ .00000$ & -1.36845 & -0.71503 & -3.93249 & 5. 97637 \\
\hline DE & 64.01799 & 2.29204 & 0.00000 & -1.62765 & -0.71503 & -1.76642 & 6.97637 \\
\hline $\mathbf{s x H}$ & 64.16799 & 2.29205 & 0.00000 & -1.73490 & -0.71503 & -1.11687 & 2.6853B \\
\hline $\mathbf{8 x h}$ & 64.31799 & 2.29205 & 0.00000 & -1.84216 & -0.71503 & -0.96081 & -0.60461 \\
\hline D5 & 54.68049 & 2.29205 & 0.00000 & -2.10136 & -0.71603 & -1.17998 & -0.50461 \\
\hline QFLH & 64.87049 & 2.23468 & -0.60133 & -2.12636 & 0,45044 & -1.20723 & 0.31 .637 \\
\hline QFLH & 55.06049 & 2.06545 & -1.17256 & -1.93352 & 1.56338 & -1.05267 & 1.19154 \\
\hline D2 & 65.52249 & 1.62373 & -1.17266 & -1.21124 & 1.56338 & -0.51217 & 1.19154 \\
\hline EDGE & 65.62249 & 1.52373 & -0.96001 & -1.24345 & -0.12438 & -0.54438 & -0.38872 \\
\hline B & E6.08249 & 1.09786 & -0.66134 & -1.17877 & 0.27967 & -0.63249 & 0.00611 \\
\hline EDGE & 56.08249 & 1.09786 & $-0,40821$ & - i. 16206 & 0.28906 & -0.61577 & 0.09380 \\
\hline D2 & 66.64449 & 0.90925 & -0.40821 & -1.02850 & 0.28908 & -0.67243 & $0.09>80$ \\
\hline SU & 56.84449 & 0.78679 & -0.40821 & -0.94178 & 0.28906 & -0.40414 & c. 84529 \\
\hline
\end{tabular}




$\begin{array}{lrrrrrrr}\text { D3 } & 58.50249 & 0.10998 & -0.40821 & -0.46262 & 0.28906 & 1.22947 & 0.98529 \\ \text { EDGE } & 58.50249 & 0.10998 & -0.39287 & -0.4628 B & 0.17646 & 1.22930 & 1.10869 \\ \text { B } & 59.06249 & 0.00000 & 0.00000 & -0.46576 & -0.19780 & 1.76068 & 0.743 \pi 6 \\ \text { BDGE } & 59.06249 & 0.00000 & 0.00000 & -0.46576 & -0.26277 & 1.76068 & 0.98766 \\ \text { D2 } & 59.62449 & 0.00000 & 0.00000 & -0.58716 & -0.26277 & 2.20698 & 0.98766 \\ \text { GFWH } & 59.71449 & 0.00000 & 0.00000 & -0.62499 & -0.13407 & 2.34916 & 0.50393 \\ \text { QFTH } & 59.90449 & 0.00000 & 0.00000 & -0.63777 & 0.00000 & 2.39719 & 0.00000 \\ \text { D1 } & 62.83299 & 0.00000 & 0.00000 & -0.63777 & 0.00000 & 2.39719 & 0.00090\end{array}$


Dispersion function $D_{0}$ and $D_{1}$ for $E$ P A

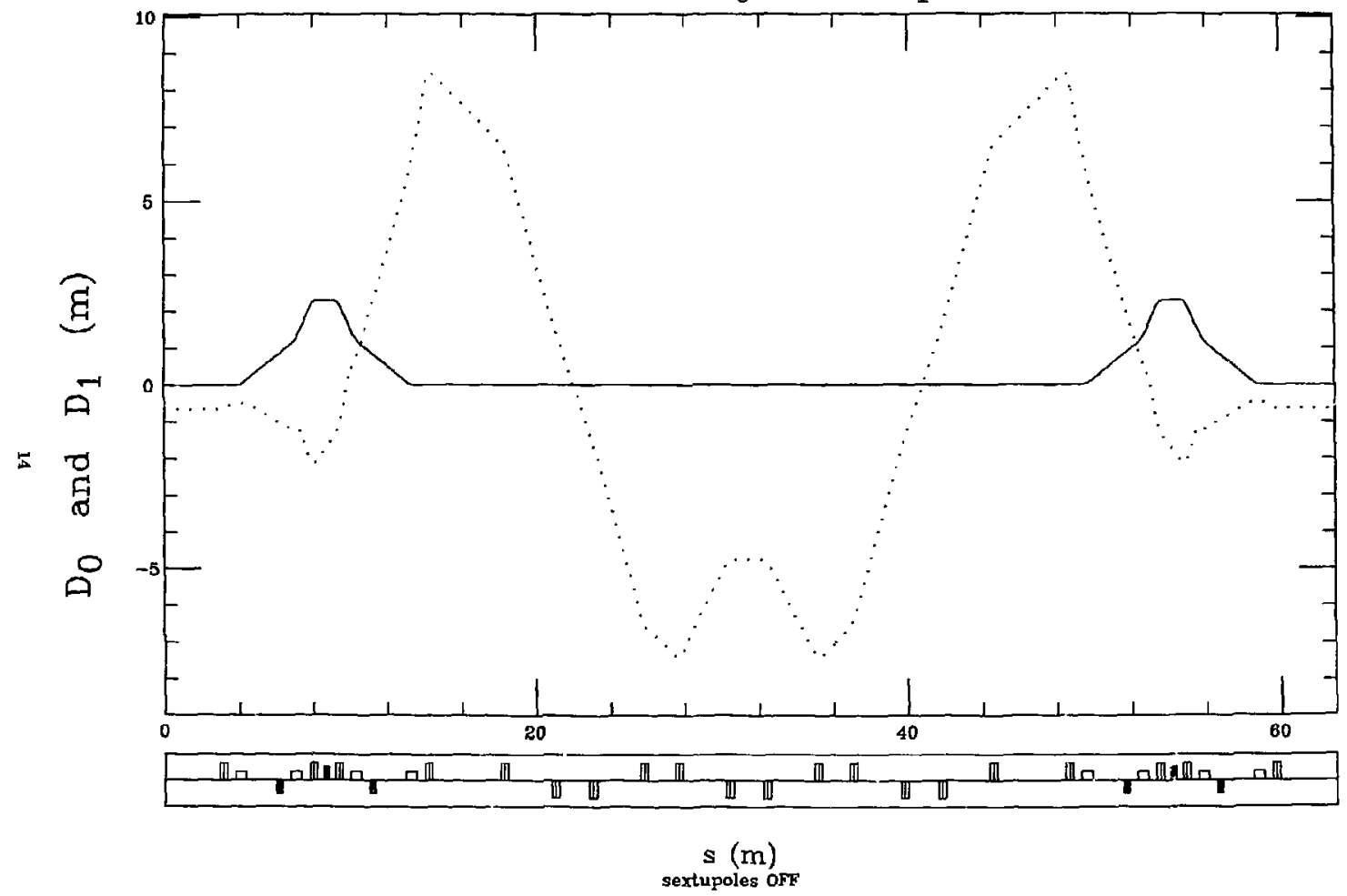

FIGURE 1 


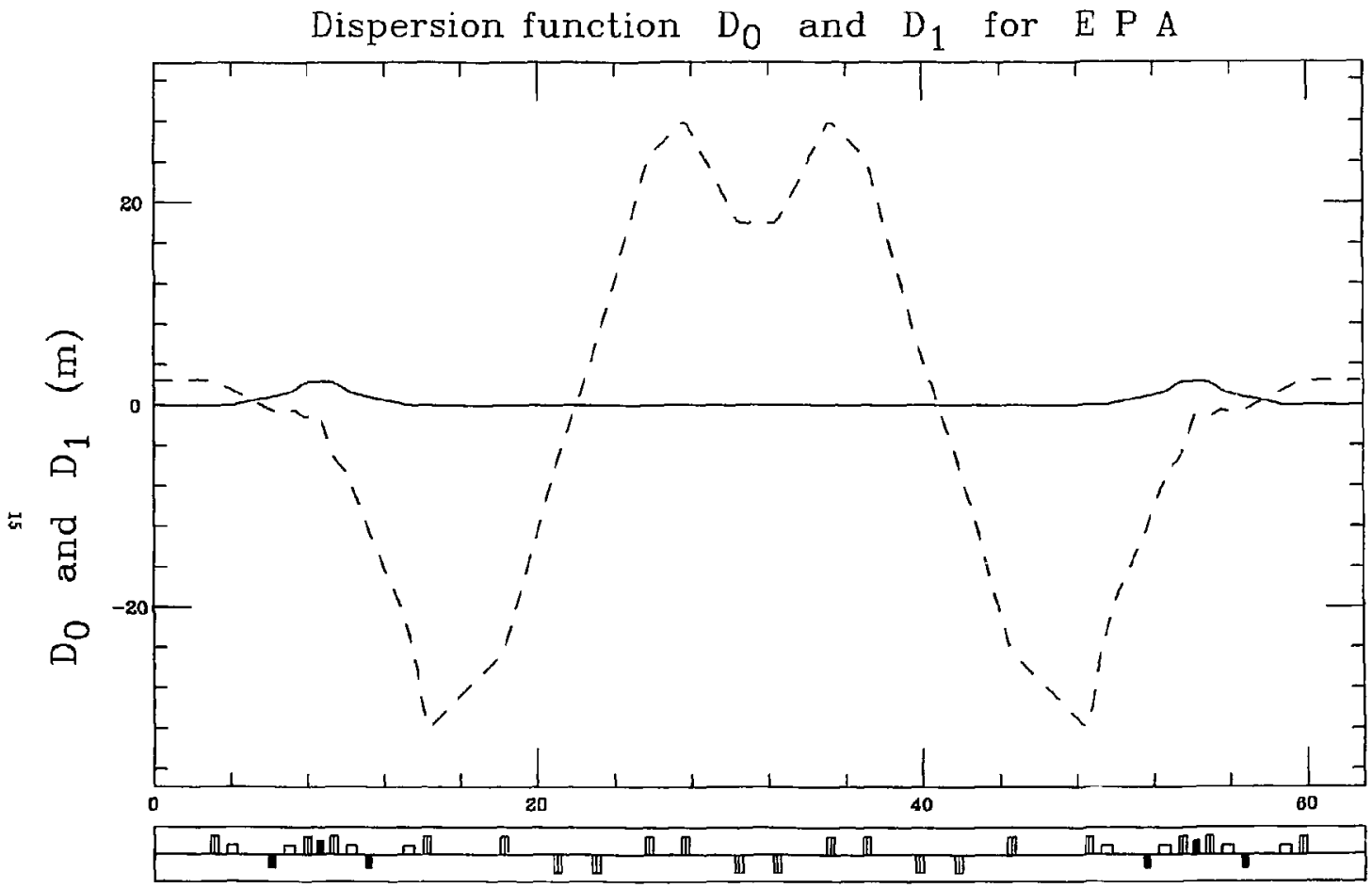

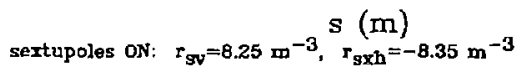

FIGURE 2 


\section{References}

1. F. C. Iselin, "The MAD Program," CERN-LEP/TH/83-30.

2. R. V. Servranckx and K. L. Brown, "Users Guide to the Program DIMAT," SLAC Report 270 UC-28 (1984).

3. K. L. Brown and R. V. Servranckx $x_{1}$ First and Second Order Charged Particle Optics," SLAC-PUB 3981.

4. B. Autin, "Simultaneous Correction of Chronaticity and Orbit Dispersion in arong Foerssing Machine, ${ }^{*} X$ Ith Int. Conf. on High Energy Accelerators, CERN, 7-12 July 1980.

5. H. Wiedemann, "Chromaticity Correction in Large Storage Rings," PEP Note 220.

6. F. T. Cole, Nonlinear Thansformation in action-angle variables,"TM 179 (FNAL).

7. E. D. Courant and H. S. Suyder, "Theory of the Alternating Gradient Synchrotron," Ann. Phys. 3, 1-48 (1958).

8. M. Donald, "Chromaticity correction in circular accelerator rings," PEP Note 221.

9. J. P. Delahaye and A. Krusehe, The Lattice Design of the LEP Electron Positron Accumulator (EPA), " IEEE Trans. on Nucl. Science, NS 30, NO.4, 2050 (A ugust 1983).

10. M. D. Woodley, M. J. Lee, J. Jäger and A. S. King, COontrol of Machine Functions or Transport Systems," SLAC-PUB 3086 (1983)

11. W. Hardt, J. Jäger, D. Möhl, *A General Analytical Expression for the Ohrometicity of Accelerator Rings," PS/LEAR/Note 82-5. 


\section{APPENDIX I}

Gontribution of the different elements to the perivdic solution of the Diepersion functions

According to the equations (3.11) the periodic solutions of the Dispersion functions are completely determined by the two integrals

$$
\int_{n}^{y} f_{i}(\sigma) R_{12}(\sigma) d \sigma \quad \text { and } \quad \int_{0}^{y} f_{i}(\sigma) R_{13}(\sigma) d \sigma
$$

with $f_{i}$ defined in eq. (3.2):

$$
\begin{aligned}
& f_{0}=h, \\
& f_{1}=-h+\left(2 h^{2}-k+h^{\prime} D_{0}^{\prime}\right) D_{0}+\left(2 h k-h^{3}+\frac{1}{2} r\right) D_{0}^{2}+\frac{1}{2} h D_{0}^{2} .
\end{aligned}
$$

Assuming hard eige approximation, where ths magnetic field rises abruptly from nero outside the magnet to a constant value inside it, the two integrals above can be computod analytically for different elements.

\section{COMBINED FUNCTION BENDING MAGNET WITE SEXTUPOLES INCLUDED}

In this case the corresponding transfer matrix elements are

$$
\begin{aligned}
& R_{11}(\sigma)=C(\sigma), \quad R_{12}(\sigma)=S(\sigma), \quad R_{13}(\sigma)=\frac{h}{h}[1-C(\sigma)], \\
& R_{21}(\sigma)=-K S(\sigma), \quad R_{22}(\sigma)=C(\sigma), \quad R_{23}(\sigma)=h S(\sigma),
\end{aligned}
$$

with the abreviations

$$
\begin{array}{ll}
K=h^{2}-k>0: & C(\sigma)=\cos (\sqrt{K} \sigma), \quad S(\sigma)=\frac{\sin (\sqrt{K} \sigma)}{\sqrt{K}} \\
K=h^{2}-k<0: & C(\sigma)=\cosh (\sqrt{|K|} \mid \sigma), \quad S(\sigma)=\frac{\sinh (\sqrt{|K| \sigma)}}{\sqrt{|K|}} .
\end{array}
$$

The Dispersion function $D_{0}$ is easily deduced from the first order transfer matrix but can 
also be determined analytically using eqs. (3.11), since

$$
\begin{aligned}
& \int_{0}^{l} f_{0}(\sigma) R_{11}(\sigma) d \sigma=h s \\
& \int_{0}^{1} f_{0}(\sigma) R_{1 g}(\sigma) d \sigma=-\frac{h}{K}(C-1)
\end{aligned}
$$

with the notation $C \equiv C(l)$ and $S \equiv S(i)$.

To calculate the perturbation $D_{11}$ one replaces, in eq. (3.2), the function $D_{0}(\sigma)$ by ita values at the beginning of that element (index 6 ) and one obtains

$$
\begin{aligned}
f_{1}(\sigma)=-h & +\left(2 h^{2}-k\right) D_{0}(\sigma)+\left(2 h k-h^{3}+\frac{1}{2} r\right) D_{0}(\sigma)^{2}+\frac{1}{2} h D_{0}^{\prime}(\sigma)^{2} \\
=-h & +\left(2 h^{2}-k\right)\left[R_{11} D_{0 b}+R_{12} D_{0 b}^{\prime}+R_{1 s}\right] \\
& +\left(2 h k-h^{3}+\frac{1}{2} r\right)\left[R_{11} D_{0 b}+R_{12} D_{0 b}^{\prime}+R_{1 s}\right]^{2} \\
& +\frac{1}{2} h\left[R_{21} D_{0 b}+R_{22} D_{0 b}^{\prime}+R_{23}\right]^{2}
\end{aligned}
$$

Integration leads to

$$
\begin{aligned}
\int_{0}^{1} f_{1}(\sigma) R_{11}(\sigma) d \sigma= & -h S+\left[\left(D_{0 b}-\frac{h}{K}\right)(l+S C)+S^{2} D_{0 b}^{\prime}\right]\left(\frac{k}{2}+\frac{k^{2}}{K}+\frac{r h}{2 K}\right) \\
& +\frac{1}{3} h D_{0 b}^{\prime}\left(D_{0 b}-\frac{h}{K}\right)\left(C^{3}-1\right)\left[1-\frac{1}{\bar{h}}\left(4 k+\frac{r}{\bar{h}}-2 h^{2}\right)\right] \\
& +\frac{1}{3} h S\left(D_{0 b}-\frac{h}{K}\right)^{2}\left[\left(2 k+\frac{r}{2 h}-h^{2}\right)\left(2+C^{2}\right)+\frac{1}{2} K^{2} S^{2}\right] \\
& +\frac{1}{2} h S D_{0 b}^{2}{ }^{2}\left[C^{2}+\frac{1}{3} S^{2}\left(2 k+\frac{r}{h}\right)\right]+S \frac{h}{K}\left(h^{2}+\frac{k^{2}}{K}+\frac{r h}{2 K}\right)
\end{aligned}
$$

and

$$
\begin{aligned}
\int_{0}^{l} f_{1}(\sigma) R_{12}(\sigma) d \sigma= & \frac{h}{K}\{C-1)+\left[\left(D_{0 b}-\frac{h}{K}\right) s^{2}+\frac{1}{K}(l-S C) D_{0 b}^{\prime}\right]\left(\frac{k}{2}+\frac{k^{2}}{K}+\frac{r h}{2 K}\right) \\
& +\frac{1}{3} h S^{3} D_{0 b}^{\prime}\left(D_{0 b}-\frac{h}{K}\right)\left(4 k-2 h^{2}-K+\frac{r}{h}\right)-\frac{h}{K^{2}}(C-1)\left(h^{2}+\frac{k^{2}}{\bar{K}}+\frac{r h}{2 K}\right) \\
& +\frac{1}{3} h\left(D_{0 b}-\frac{h}{K}\right)^{2}\left[\frac{1}{K}\left(1-C^{3}\right)\left(2 k-h^{2}+\frac{r}{2 h}\right)+1+\frac{1}{2} C^{3}-\frac{3}{2} C\right] \\
& +\frac{1}{3} D_{0 b}^{\prime 2} \frac{h}{K}\left[\frac{1}{2}\left(1-C^{3}\right)+\frac{1}{K}\left(2+C^{3}-3 C\right)\left(2 k-h^{2}+\frac{r}{2 h}\right)\right]
\end{aligned}
$$


In the particular case of $K=h^{2}-k \equiv 0$, these equations simplify to

$$
\begin{aligned}
& \int_{0}^{l} f_{1}(\sigma) d \sigma=l\left\{-h+h D_{0 b}\left[h+\frac{l^{2}}{3}\left(h^{3}+\frac{1}{2} r\right)\right]+h l D_{0 b}^{\prime}\left[h+\frac{l^{2}}{4}\left(h^{3}+\frac{1}{2} r\right)\right]\right. \\
& +1 D_{0 b} D_{0 b}^{\prime}\left(h^{3}+\frac{1}{2} r\right)+D_{0 b}^{2}\left(h^{3}+\frac{1}{2} r\right) \\
& \left.+D_{0 b}^{\prime} *\left[\frac{h}{2}+\frac{l^{2}}{3}\left(h^{3}+\frac{1}{2} r\right)\right]+h^{2} l^{2}\left[\frac{h}{3}+\frac{h^{2}}{20}\left(h^{3}+\frac{1}{2} r\right)\right]\right\} \text {. } \\
& \int_{0}^{1} f_{1}(\sigma) \sigma r_{1,}=l^{2}\left\{-\frac{h}{2}+\frac{1}{2} h D_{0 b}\left[h+\frac{l^{2}}{2}\left(h^{3}+\frac{1}{2} r\right)\right]+h l D_{0 b}^{\prime}\left[\frac{2}{3} h+\frac{l^{2}}{5}\left(h^{3}+\frac{1}{2} r\right)\right]\right. \\
& +\frac{2}{3} l D_{0 b} D_{0 b}^{\prime}\left(h^{3}+\frac{1}{2} r\right)+\frac{1}{2} D_{0 b}^{2}\left(h^{3}+\frac{1}{2} r\right) \\
& \left.+\frac{1}{4} D_{O b}^{\prime 2}\left[h+l^{2}\left(h^{3}+\frac{1}{2} r\right)\right]+\frac{1}{4} h^{2} l_{b}^{2}\left[h+\frac{l^{2}}{6}\left(h^{3}+\frac{1}{2} r\right)\right]\right\} \text {. }
\end{aligned}
$$

The expressions corresponding to elementary elements can then easily be deduced by cancelling the relevant parameters in the general expressions of the integrals and one gets:

\section{Pure drift:}

$$
\begin{aligned}
& \int_{0}^{1} f_{1}(\sigma) R_{11}(\sigma) d \sigma=0 \\
& \int_{0}^{1} f_{1}(\sigma) R_{12}(\sigma) d \sigma=0
\end{aligned}
$$

Pure sextupole:

$$
\begin{aligned}
& \int_{0}^{l} f_{1}(\sigma) R_{11}(\sigma) d \sigma=\frac{1}{2} r l\left[D_{0 b}^{2}+l D_{0 b} D_{0 b}^{\prime}+\frac{1}{9} l^{2} D_{0 b}^{2}\right] \\
& \int_{0}^{l} f_{1}(\sigma) R_{12}(\sigma) d \sigma=\frac{1}{2} r l^{2}\left[\frac{1}{2} D_{0 b}^{2}+\frac{2}{3} l D_{0 b} D_{0 b}^{\prime}+\frac{1}{4} l^{2} D_{0 b}^{2}\right]
\end{aligned}
$$


Pure quadrupole:

$$
\begin{aligned}
& \int_{0}^{t} f_{1}(\sigma) R_{11}(\sigma) d \sigma=-\frac{1}{2} k\left[(l+S C) D_{0 b}+S^{2} D_{0 b}^{t}\right] \\
& \int_{0}^{l} f_{1}(\sigma) R_{1 A}(\sigma) d \sigma=-\frac{1}{2}\left[k S^{2} D_{0 b}-(l-s C) D_{0 b}^{\prime}\right]
\end{aligned}
$$

\section{EDGES}

With $\theta(\sigma)$ as the angle between the pole face of the element and the particle trajectory the transfer matrix elements are defined as

$$
\begin{array}{lll}
R_{11}=1, & R_{12}=\sigma, & R_{13}=0, \\
R_{21}=\int h^{\prime} \tan d \sigma, & R_{22}=1, & R_{23}=0,
\end{array}
$$

since the variation $\boldsymbol{h}^{\prime}$ of the curvature cannot be neglected. Moreover, for magnetic elements with inclined houndaries whose entrance or exit faces are not normal to the design trajectory, the local quadrupole $k(\sigma)$ and sextupole $r(\sigma)$ components acting on a particle passing through the fringe field of a magnetic elemant along the design trajectory are given by ${ }^{(11)}$

$$
\begin{aligned}
& k(\sigma)=h^{\prime} \tan \theta, \\
& r(\sigma)=-2 k_{m}^{\prime} \tan \theta \mp h^{\prime \prime} \tan ^{2} \theta+h h^{\prime} \tan ^{3} \theta,
\end{aligned}
$$

where

$$
\theta=\theta_{0}-\int h d a
$$

The sign convention for the entrance (exit) angle $\theta_{e}$ is the same as in ${ }^{(9)}$; the first and second derivatives with respect to the aximuth $\sigma$ are denoted by ' and " respectively and $k_{\mathrm{m}}$ is the quadrupole component of the element. The upper sign corresponds to the entrance edge, the lower sign to an exit edge. Introducing these relations for the quadrupole and sextupole fields into the equation for $f_{1}(\sigma)$ defined in eq. (3.2), integrating over the edgea and applying the "hard 
edge" approximation $(s \rightarrow 0)$ gives

$$
\begin{array}{r}
\lim _{g \rightarrow 0} \int_{0} f_{1}(\sigma) R_{11}(\sigma) d \sigma=\lim _{\theta \rightarrow 0} \int_{0}^{\infty} f_{1}(\sigma) d \sigma= \pm h\left(D_{0}^{\prime}\right)_{e} D_{0 e}\left(1+\tan ^{2} \theta_{e}\right)-h D_{0 \varepsilon}\left(1-h D_{0 e}\right) \tan \theta_{e} \\
-k D_{0 e}^{2} \tan \theta_{e}-\frac{1}{2} h^{2} D_{0 \sigma}^{\alpha}\left(1+\frac{1}{2} \tan ^{2} \theta_{\sigma}\right) \tan \theta_{e}
\end{array}
$$

where the index " $m$ " has been omitted. The index ${ }^{k} e^{n}$ denotes either the entrance of the entrance edge or the exit of the exit edge. After introducing

$$
\left\langle D_{0}^{\prime}\right\rangle_{e}=D_{0 e}^{\prime} \pm \frac{1}{2} h D_{0 e} \tan \theta_{e}
$$

the relation for the entrance (upper sign) and for the exit edge (lower sign) is

$$
\begin{aligned}
\lim _{e \rightarrow 0} \int_{0} f_{1}\left\{\sigma \mid R_{11}(\sigma) d \sigma= \pm h D_{0 \varepsilon}^{\prime} D_{0 e}\left(1+\tan ^{2} \theta_{e}\right)-h D_{0 e}\left(1-h D_{0 e}\right) \tan \theta_{e}-k D_{0 e}^{2} \tan \theta_{e}\right. \\
+\frac{1}{4} h^{2} D_{0 e}^{2} \tan ^{3} \theta_{e}
\end{aligned}
$$

Similarily, one obtains

$$
\lim _{\sigma \rightarrow 0} \int_{0}^{i} f_{L}(\sigma) R_{12}(\sigma) d \sigma=\lim _{b \rightarrow 0} \int_{0}^{\infty} \sigma f_{1}(\sigma) d \sigma= \pm \frac{1}{2} h D_{0 e}^{2} \tan ^{2} \theta_{e} .
$$




\section{APPENDIX II}

Change of the Dispersion function $D_{1}$ due to different elements

Introducing into eqs. (4.2) the expreseions for the integrels derived in APPENDIX I and rearranging the different terms, one obtaine the relationa for the tranaport of the function $D_{1}$ through the different machine elements.

\section{COMBINED FUNCTION BENDING MAGNET WITH SEXTUPOLES INCLUDED}

$$
\begin{aligned}
D_{1}(l)= & D_{1 b} C+D_{2 b}^{\prime} S-\frac{h}{K}(1-C) \\
+ & {\left[\left(D_{0 b}-\frac{h}{K}\right) I S+\frac{1}{K}(S-l C) D_{0 b}^{\prime}\right]\left(\frac{k}{2}+\frac{k^{2}}{K}+\frac{r h}{2 K}\right) } \\
+ & \frac{1}{3} S D_{0 b}^{p}\left(D_{0 b}-\frac{h}{K}\right)(1-C)\left[\frac{1}{K}\left(4 h k+r-2 h^{3}\right)-h\right] \\
+ & \frac{1}{3}\left(D_{0 b}-\frac{h}{K}\right)^{2}\left[\frac{1}{K}\left(2 h k+\frac{1}{2} r-h^{3}\right)\left(2-C-C^{2}\right)+\frac{1}{2} h(1-C)^{2}\right] \\
+ & \frac{1}{3} D_{0 b}^{\prime} \frac{h}{K}\left\{C\left(\frac{3}{2} C-\frac{1}{2}-C^{3}\right)+\frac{1}{K}\left[k\left(1+4 C^{2}-4 C-C\right)+h^{2} C\left(2+C^{3}-3 C\right)\right]\right. \\
& \left.+\frac{r}{2 h K}(1-C)^{2}\right\}+\frac{h}{K^{2}}\left(h^{2}+\frac{k^{2}}{K}+\frac{r h}{2 K}\right)(1-C) \\
& +\left[\left(D_{0 b}-\frac{h}{K}\right)(l C+S)+S\left(D_{0 b}^{\prime}\right]\left(\frac{k}{2}+\frac{k^{2}}{K}+\frac{r h}{2 K}\right)\right. \\
& +\frac{1}{3} D_{0 b}^{\prime}\left(D_{0 b}-\frac{h}{K}\right)\left(C+1-2 C^{2}\right)\left[\frac{1}{K}\left(4 h k+r-2 h^{3}\right)-h\right] \\
& +\frac{1}{3} h S\left(D_{0 b}-\frac{h}{K}\right)^{2}\left[\left(2 k+\frac{r}{2 h}-h^{2}\right)(2 C+1)+K(1-C)\right] \\
& +\frac{1}{3} h S D_{0 b}^{\prime}\left\{C^{3}+\frac{1}{2}+\frac{1}{\bar{K}}\left(k\left(4+C^{3}-5 C\right)-h^{2}\left(2+C^{3}-3 C\right)\right]+\frac{r}{h K}(1-C)\right\} \\
& +S \frac{h}{K}\left(h^{2}+\frac{k^{2}}{K}+\frac{r h}{2 K}\right) \\
D_{1}^{\prime}(l)= & -D_{1 b} K S+D_{1 b}^{\prime} C-h S
\end{aligned}
$$


In the particular case of $K=h^{2}-k \equiv 0$, these equations simplify to

$$
\begin{aligned}
& D_{1}(I)=D_{1 b}+I D_{1 b}^{\prime}-\frac{1}{2} h l^{2} \\
& +l^{2}\left\{\frac{1}{2} h D_{0 b}\left[h+\frac{l^{2}}{6}\left(h^{3}+\frac{1}{2} r\right)\right]+h l D_{0 b}^{\prime}\left[\frac{h}{3}+\frac{l^{2}}{20}\left(h^{3}+\frac{1}{2} r\right)\right]\right. \\
& +\frac{1}{3} l D_{0 b} D_{0 b}^{\prime}\left(h^{3}+\frac{1}{2} r\right)+\frac{1}{2} D_{0 b}^{2}\left(h^{3}+\frac{1}{2} r\right) \\
& \left.+\frac{1}{4} D_{0 b}^{2}\left[h+\frac{l^{2}}{3}\left(h^{3}+\frac{1}{2} r\right)\right]+\frac{1}{12} h^{2} l^{2}\left[h+\frac{l^{2}}{10}\left(h^{3}+\frac{1}{2} r\right)\right]\right\} \\
& D_{1}^{\prime}(l)=D_{1 b}^{\prime}-h l \\
& +l\left\{h D_{0 b}\left[h+\frac{l^{2}}{3}\left(h^{3}+\frac{1}{2} r\right)\right]+h l D_{0 b}^{\prime}\left[h+\frac{l^{2}}{4}\left(h^{3}+\frac{1}{2} r\right)\right]\right. \\
& +l D_{0 b} D_{0 b}^{\prime}\left(h^{3}+\frac{1}{2} r\right)+D_{0 b}^{2}\left(h^{3}+\frac{1}{2} r\right) \\
& \left.+D_{0 b}^{\prime 2}\left[\frac{h}{2}+\frac{l^{2}}{3}\left(h^{3}+\frac{1}{2} r\right)\right]+h^{2} l^{2}\left[\frac{h}{3}+\frac{l^{2}}{20}\left(h^{3}+\frac{1}{2} r\right)\right]\right\}
\end{aligned}
$$

One can easily deduce the expressions corresponding to elementary elemente by cancelling the relevant parameters:

Pure drift:

$$
\begin{aligned}
& D_{1}(l)=D_{1 b}+D_{1 b}^{\prime} l \\
& D_{1}^{\prime}(l)=D_{1 b}^{\prime}
\end{aligned}
$$

Pure sextupole:

$$
\begin{aligned}
& D_{1}(l)=D_{1 b}+D_{1 b}^{t} l+\frac{1}{2} r l^{2}\left(\frac{1}{2} D_{0 b}^{2}+\frac{1}{3} D_{0 b} D_{0 b}^{\prime} l+\frac{1}{12} D_{0 b}^{\prime 2} l^{2}\right) \\
& D_{1}^{\prime}(l)=D_{1 b}^{\prime}+\frac{1}{2} r l\left(D_{0 b}^{2}+D_{0 b} D_{0 b}^{\prime} l+\frac{1}{3} D_{0 b}^{\prime 2} l^{2}\right)
\end{aligned}
$$

Pure quadrupole:

$$
\begin{aligned}
& D_{1}(l)=D_{1 b} C+D_{1 b}^{\prime} S-\frac{1}{2}\left[k D_{0 b} l S-(S-l C) D_{0 b}^{\prime}\right] \\
& \left.D_{1}^{\prime}(l)=D_{1 b} k S+D_{1 b}^{\prime} C-\frac{1}{2} k \mid(S+l C) D_{0 b}+D_{0 b}^{\prime} l S\right]
\end{aligned}
$$




\section{EDGES}

Entrance edge : entrance angle $\theta_{1}$

$\left(D_{1}, D_{1}^{t}\right.$ at the end of the entrance edge defined by the values at the beginning, index " $b^{n}$ )

$$
\begin{aligned}
D_{1}= & D_{1 b}-\frac{1}{2} h D_{0 b}^{2} \tan ^{2} \theta_{1} \\
D_{1}^{\prime}= & D_{1 b} h \tan \theta_{1}+D_{1 b}^{\prime} \\
& \quad+h D_{0 b}^{\prime} D_{0 b}\left(1+\tan ^{2} \theta_{1}\right)-h D_{0 b}\left(1-h D_{1 b}\right) \tan \theta_{1}-h D_{0 b}^{2} \tan \theta_{1}
\end{aligned}
$$

Exit edge : exit angle $\theta_{2}$

$\left(D_{1}, D_{1}^{\prime}\right.$ at the end of the exit edge defined by the valnes at the beginning, index " $b$ ")

$$
\begin{aligned}
D_{1}= & D_{13}+ \\
D_{1}^{\prime}= & D_{1 b} h \tan \theta_{2}+D_{1 b}^{2} \tan ^{2} \theta_{2} \\
& -h D_{0 b}^{\prime} D_{0 b}\left(1+\tan ^{2} \theta_{2}\right)-h D_{0 b} \tan \theta_{3}-k D_{0 b}^{2} \tan \theta_{2}-\frac{1}{2} h^{2} D_{0 b}^{2} \tan ^{3} \theta_{3}
\end{aligned}
$$

\section{DISCLAIMER}

This report was propared as an aceount of work sponsored by an agency of the United States Government. Neither the United States Government nor any agency thereof, nor any of their employes, makes any warranty, express or implied, of assumes any legal liability or responsibility for the accuracy, completeness, of usefulness of any info:mation, apparatus, product, or process disclosed, of teptestents that its use would not infringe privately owned rights. Reference hertin to any specific commercial product, process, or service by trade name, trademark, manufactures, or otherwike does not necessarily constitute or imply its endorsement, recommendation, or favoring by the United States Government or any agency thereof. The views and opinions of tuthors expressed hertin do not necessarily state or reflect thost of the United States Governmtent or any agency thereof. 